\title{
Special Issue: Biomolecular Motors and Motor Assemblies
}

\author{
Henry Hess ${ }^{1}$ and Jung-Chi LiaO ${ }^{2}$ \\ ${ }^{1}$ Department of Biomedical Engineering, Columbia University, New York, NY, USA; and ${ }^{2}$ Department of Mechanical \\ Engineering, Columbia University, New York, NY, USA \\ published online 3 January 2013
}

Since the first in vitro observations of movement driven by molecular motors by Sheetz and Spudich in 1983, 30 years of work have brought us toward a better foundation in understanding how molecular motors play roles in different organisms and how they can be used in biotechnology applications. Recent advances in this area were highlighted in a session "Biomolecular Motors and Force Generation" at the first US Association for Computational Mechanics Thematic Conference on Multiscale Methods and Validation in Medicine and Biology held in February 2012. This event featuring 15 oral presentations, including a keynote speech by George Oster, generated fruitful discussions among researchers in the field and led us to conceive of this special issue.

A majority of biomolecular motors are ATPdependent, coupling ATP hydrolysis energy to mechanical work. These motors include cytoskeletal myosin, kinesin, and dynein, as well as nucleic acidbased translocases, ATP-dependent chaperones, and ATP synthase. Each molecular motor generates forward movement along its specific substrate (myosin on actin, kinesin and dynein on microtubule, ATPdependent chaperones on peptides, nucleic acid-based translocases on DNA or RNA, and ATP synthase on the central shaft). The motor-substrate interactions and their corresponding functions, and the interactions between individual motors are one focus of this special issue.

For the interaction between myosin and actin filaments, Yasuhiro Inoue and Taiji Adachi illustrate how actin-myosin catch bonds affect actomyosin aggregate formation. Using computer simulations they found that the catch-bond behavior facilitates the assembly of myosin in a concentration dependent manner. The actin-myosin interaction thus can influence the properties of actin-myosin networks at a larger scale which in turn affect cellular morphogenesis. In muscle, the

Address correspondence to Henry Hess, Department of Biomedical Engineering, Columbia University, New York, NY, USA. Electronic mail: hh2374@columbia.edu,jcliao@columbia.edu interaction between a myosin motor and an actin filament can be affected by the binding of neighboring myosins to the actin filament. This cooperative effect is not captured by classical models based on linear partial differential equations. Sam Walcott's contribution is a new model which includes these cooperative effects and is an exciting step towards a complete multi-scale description of muscle contraction. Travis Stewart and his colleagues from the research group of Josh Baker describe an experimental study which shows that the details of actin-myosin interactions affect muscle shortening speeds not only by defining the detachment rates but also by influencing the attachment kinetics.

In contrast to actomyosin's cooperativity, kinesins often hinder each other except under high loads, and the detailed mechanism behind this load-dependent cooperativity is unclear. Uppulury et al. used a stochastic model to identify biochemical and structural parameters affecting this negative cooperativity. This contribution continues the advances made by Anatoly Kolomeisky's and Michael Diehl's groups in the understanding of kinesin-driven intracellular transport. The behavior of two elastically coupled motors is studied theoretically by Florian Berger et al., who derived explicit results to describe the general dynamics of pairs of biomolecular motors. These results enable the prediction of distinct behaviors of different motor species and build upon prior work by Reinhard Lipowsky and Stefan Klumpp.

A second focus of this special issue is kinesin's counterpart in microtubule-associated transport, the dynein motor. Recently solved crystal structures of dynein facilitate further analysis of dynein activities. Dynein belongs to the AAA + protein superfamily, forming a ring-shaped structure with multiple catalytic sites. Wonmuk Hwang and Matthew Lang used a coarse-grained model to study dynein as well as ClpX and HslU, two ATP-dependent peptide translocases. They found that nucleotide-free and nucleotide occupied subunits work together to generate force while enabling a variety of motor designs. Nandini Shekar and Jun $\mathrm{Wu}$ review the accumulated knowledge and its 
implications about the role of dynein-generated forces on intracellular organization under the guidance of Richard Dickinson and Tanmay Lele. A recurring theme is that motors do not only generate forces but also cause friction, which plays an important role in the observed behavior of the system. Bhavik Nathwani, Tony Yang, and Jung-Chi Liao survey the role of both dynein and kinesin in primary cilia and discuss how recent advances in superresolution imaging can aid our understanding of the motor-mediated transport in primary cilia.

Lastly, two contributions discuss the properties of kinesin-driven transport of microtubules in in vitro experiments aiming at the assembly of nanocomposites and the rapid capture of analytes from a solution. Haiqing Liu and George Bachand describe their experimental results investigating for the first time the effect of spatial constraints on the assembly of microtubule/quantum dot structures. Takahiro Nitta and Henry Hess simulate the aggregation of analytes by kinesin-driven microtubule shuttles which is critically affected by the multiscale nature of the gliding trajectories.

All together, the contributions highlight the interplay between experiments, computer simulations, and theoretical models in the study of these complex systems as well as the importance of thoughtful reviews of the accumulating evidence. As the editors of this special issue we would like to thank Ed Guo for his encouragement and assistance in his role as co-Editor in Chief of the journal, and most importantly we would like to thank the reviewers whose expertise and judgment greatly contributed to the success of this endeavor. 